\title{
Individualized therapy for metastatic renal cell carcinoma
}

\author{
Bambang T. Atmaja ${ }^{1}$, Izabelle Wood ${ }^{1}$, Suyanto Suyanto ${ }^{1}$, Paramvir Sawhney ${ }^{2}$, Agnieszka Michael $^{1}$, Hardev \\ Pandha ${ }^{1}$ \\ 'St Luke's Cancer Centre, Royal Surrey County Hospital, Guildford GU2 7XX, UK. \\ ${ }^{2}$ Department of Oncology, University College London Cancer Institute, London WC1E 6DD, UK. \\ Correspondence to: Dr. Bambang T. Atmaja and Dr. Izabelle Wood, St Luke's Cancer Centre, Royal Surrey County Hospital, \\ Egerton Road, Guildford GU2 7XX, UK. E-mail: bambang.atmaja@nhs.net; izabelle.wood1@nhs.net
}

How to cite this article: Atmaja BT, Wood I, Suyanto S, Sawhney P, Michael A, Pandha H. Individualized therapy for metastatic renal cell carcinoma. J Cancer Metastasis Treat 2021;7:xx. https://dx.doi.org/10.20517/2394-4722.2021.66

Received: 15 Mar 2021 First Decision: 8 May 2021 Revised: 19 May 2021 Accepted: 28 May 2021 First online: 2 Jun 2021

Academic Editor: Lucio Miele Copy Editor: Yue-Yue Zhang Production Editor: Yue-Yue Zhang

\begin{abstract}
Metastatic Renal Cell Carcinoma (mRCC) is a highly heterogeneous disease that is notoriously difficult to treat successfully. However, the discovery of novel, targeted therapies over the last decade has revolutionized its management. As the therapeutic options continue to evolve, developing a more individualized treatment strategy is of paramount importance. The International mRCC Database Consortium (IMDC) is a prognostic model that is commonly used in trials and clinical settings to risk stratify patients. This allows for optimal therapy selection on a more individual basis. However, the distinct lack of validated predictive biomarkers in mRCC renders it difficult to assess therapy response. An improved understanding of tumor biology and genetics has prompted a shift from cytokine therapy to the use of vascular endothelial growth factor (VEGF) inhibitors, tyrosine kinase Inhibitors, immune checkpoint inhibitors or combination strategies. Studies have identified some putative markers and genetic mutations as potential predictors of therapy response. Early results are promising, and there are many ongoing trials further assessing their suitability for clinical use. This review will evaluate the current treatment landscape and molecular biology of mRCC, with a specific focus on the prognostic and predictive markers available to guide treatment options and further improve patient outcomes.
\end{abstract}

Keywords: Metastatic renal cell carcinoma, IMDC, predictive biomarker, individualized therapy, immunotherapy, VEGF, prognosis 


\section{INTRODUCTION}

Renal Cell Cancer (RCC) represents 2\%-3\% of global cancer diagnoses ${ }^{[1]}$. However, its incidence in the developed world has doubled over the past half-century and is projected to increase ${ }^{[2]}$. It is the 7 th most common cancer in the UK, with around 13,100 new diagnoses each year ${ }^{[3]}$. About $30 \%$ of patients present with metastatic disease at the time of diagnosis and an additional $30 \%$ of patients undergoing curative surgery for localized RCC, will develop recurrence or metastases ${ }^{[4]}$.

The systemic treatment for mRCC has evolved substantially over the last decade owing to a better understanding of the underlying biology of RCC. The discovery of the significance of the vascular endothelial growth factor (VEGF) and mammalian target of rapamycin (mTOR) pathways has radically shifted RCC management from interferons in the mid-2000s to novel, targeted agents. More recently, several immune checkpoint inhibitors (ICIs) have joined the therapeutic options of mRCC. The spectrum of overall survival in a vastly heterogenous disease such as mRCC ranges from a few months to many years. Therefore, a risk stratification tool is of paramount importance to guide future individualized treatment decision-making. In addition, predictive biomarkers are critical for developing personalized care in oncology; examples include anti-HER2 antibody in HER2-positive breast cancer and BRAF inhibitors in BRAF mutant melanomas ${ }^{[5]}$. Unfortunately, no biomarkers currently have equivalent utility in mRCC despite the obvious dependence of this disease on the VEGF pathway.

This article will review the treatment landscape of mRCC, evaluate the available risk prognostication tools and explore potential predictive markers that may help achieve the goal of personalized systemic therapy in kidney cancer.

\section{PROGNOSTIC CLINICAL MODELS}

Appropriate treatment selection in clinical practice is facilitated by prognostic stratification. The era of VEGF-targeted therapy saw the development of the International mRCC Database Consortium (IMDC), a clinical model that integrates six variables to stratify patients with mRCC into three prognostic groups (favorable, intermediate and poor-risk) [Table 1]. It incorporates six prognostic factors that correlate independently with overall survival (OS): Karnofsky performance status score of less than $80 \%$, an interval of less than 1 year between diagnosis of RCC and initiation of treatment, corrected serum calcium level greater than $10 \mathrm{mg} / \mathrm{dL}$, hemoglobin levels below the lower limit of normal, high absolute neutrophil and platelet count. This has largely superseded the Memorial Sloan Kettering Cancer Centre (MSKCC) model, commonly used in the era of interferon therapy ${ }^{[0,7]}$. The median OS associated with each prognostic group is 43 months, 23 months, and 8 months in the favorable, intermediate and poor-risk groups, respectively ${ }^{[8]}$. The IMDC has not only been shown to profile risk using VEGF-targeted agents in the first-line setting, but also in the second and third-line settings ${ }^{[9,10]}$. Although the IMDC was specifically applicable to anti-VEGF therapy, its positive value has also been demonstrated in patients receiving single or combination immunotherapy ${ }^{[1-113]}$.

\section{THE USE OF PREDICTIVE BIOMARKERS IN METASTATIC RENAL CELL CARCINOMA}

There are various histological subtypes of renal cell carcinoma; the most common of these being clear cell RCC (ccRCC), which accounts for over $75 \%$ of diagnoses ${ }^{[14]}$. The molecular heterogeneity within each subtype has affected the success of biomarker discovery and may explain the variable responses to systemic therapies ${ }^{[5]}$. Therefore interpretation and validation of certain molecular markers will be key to further enhancing the individualized management of RCC. 
Table 1. Differences between the MSKCC and IMDC risk prognostication tools for mRCC ${ }^{[6-8]}$

\begin{tabular}{|c|c|c|}
\hline & MSKCC & IMDC \\
\hline \multicolumn{3}{|l|}{ Prognostic factors } \\
\hline Low Karnofsky performance $(<80 \%)$ & $\sqrt{ }$ & $\sqrt{ }$ \\
\hline Time from diagnosis to treatment $<1$ year & $\sqrt{ }$ & $\sqrt{ }$ \\
\hline Low haemoglobin $(<$ LLN) & $\sqrt{ }$ & $\sqrt{ }$ \\
\hline High corrected calcium (> ULN) & $\sqrt{ }$ & $\sqrt{ }$ \\
\hline High neutrophils (> ULN) & & $\sqrt{ }$ \\
\hline High platelets (> ULN) & & $\sqrt{ }$ \\
\hline High LDH (> 1.5 times ULN) & $\sqrt{ }$ & \\
\hline \multicolumn{3}{|l|}{ Entry population criteria } \\
\hline MRCC patient & Interferon as frontline therapy & First-line TKI therapy \\
\hline \multicolumn{3}{|l|}{ Median OS (by risk groups) } \\
\hline O criteria (favorable) & 29.6 months ( $95 \% \mathrm{Cl}: 20.9-37.8$ months) & 43.2 months (95\%Cl: $31.4-50.1$ months) \\
\hline 1-2 criteria (intermediate) & 13.8 months ( $95 \% \mathrm{Cl}: 12.4-15.9$ months) & 22.5 months ( $95 \% \mathrm{Cl}: 18.7-25.1$ months) \\
\hline$\geq 3$ criteria (poor) & 4.9 months ( $95 \% \mathrm{Cl}: 4.3-6.3$ months) & 7.8 months ( $95 \% \mathrm{Cl}: 6.5-9.7$ months) \\
\hline
\end{tabular}

The inactivation of von Hippel-Lindau (VHL) is the most common mutation observed in ccRCC ${ }^{[15]}$. The loss of VHL leads to hypoxia-inducible factor (HIF) accumulation, which in turn results in the overexpression of pro-angiogenic VEGF and platelet-derived growth factor (PDGF) ${ }^{[15,16]}$. Sunitinib, a tyrosine kinase Inhibitors (TKI), blocks VEGF and PDGF receptors [Figure 1] ${ }^{[17]}$; it is used as a first or second-line therapy in ccRCC. Research suggests that the loss of VHL is not sufficient to promote oncogenesis, and that mutations of epigenetic regulators such as PBRM1 and BAP1 are required to drive the development of $c \mathrm{cRCC} \mathrm{C}^{[18]}$. Studies indicate that tumors with BAP1 mutations are correlated with unfavorable outcomes in both local and metastatic disease $\mathrm{e}^{[19,20]}$.

The PI3K/AKT/mTOR pathway plays an important role in the tumorigenesis of ccRCC. The mTOR complex regulates cell growth, metabolism and angiogenesis; mutations can lead to dysregulation of these functions ${ }^{[21]}$. Everolimus and Temsirolimus are mTOR inhibitors approved for use in mRCC [Figure 1] $]^{[22]}$. Of the two known mTOR signaling pathways, namely mTORC1 and mTORC2, these therapies only block one (mTORC1), leaving the second downstream signaling of mTOR activation unopposed ${ }^{[23,24]}$. Research has identified that tumor mutations in mTOR, TSC1 or 2 (mTOR regulators) are more common in patients who clinically respond to mTOR inhibitors ${ }^{[25]}$. However, one study found that a large proportion of responders had no mTOR pathway mutation detected ${ }^{[2]}$, suggesting that these mutations cannot yet be considered as useful biomarkers.

ICIs are commonly used in the treatment of mRCC. The combination of Nivolumab, a Programmed Cell Death (PD-1) inhibitor and Ipilimumab, a Cytotoxic T-Lymphocyte-associated Antigen 4 (CTLA-4) inhibitor, is an approved first-line systemic therapy ${ }^{[26]}$. In many malignancies, Programmed Death-Ligand 1 ( $P D-L 1)$ expression has been demonstrated as a reliable predictor of therapy response; however, this is not the case in RCC. The CheckMate-025 and -214 trials recorded respectable response rates with immunotherapy regardless of $P D-L 1$ expression ${ }^{[27-29]}$. Unfortunately, its use as a potential biomarker is limited because of tumor heterogeneity and the fact that $P D-L 1$ expression can be modified by prior VEGFtargeted therapies ${ }^{[30]}$. Nevertheless, research suggests that a high level of $P D-L 1$ expression is an unfavorable prognostic factor $^{[30,31]}$. 


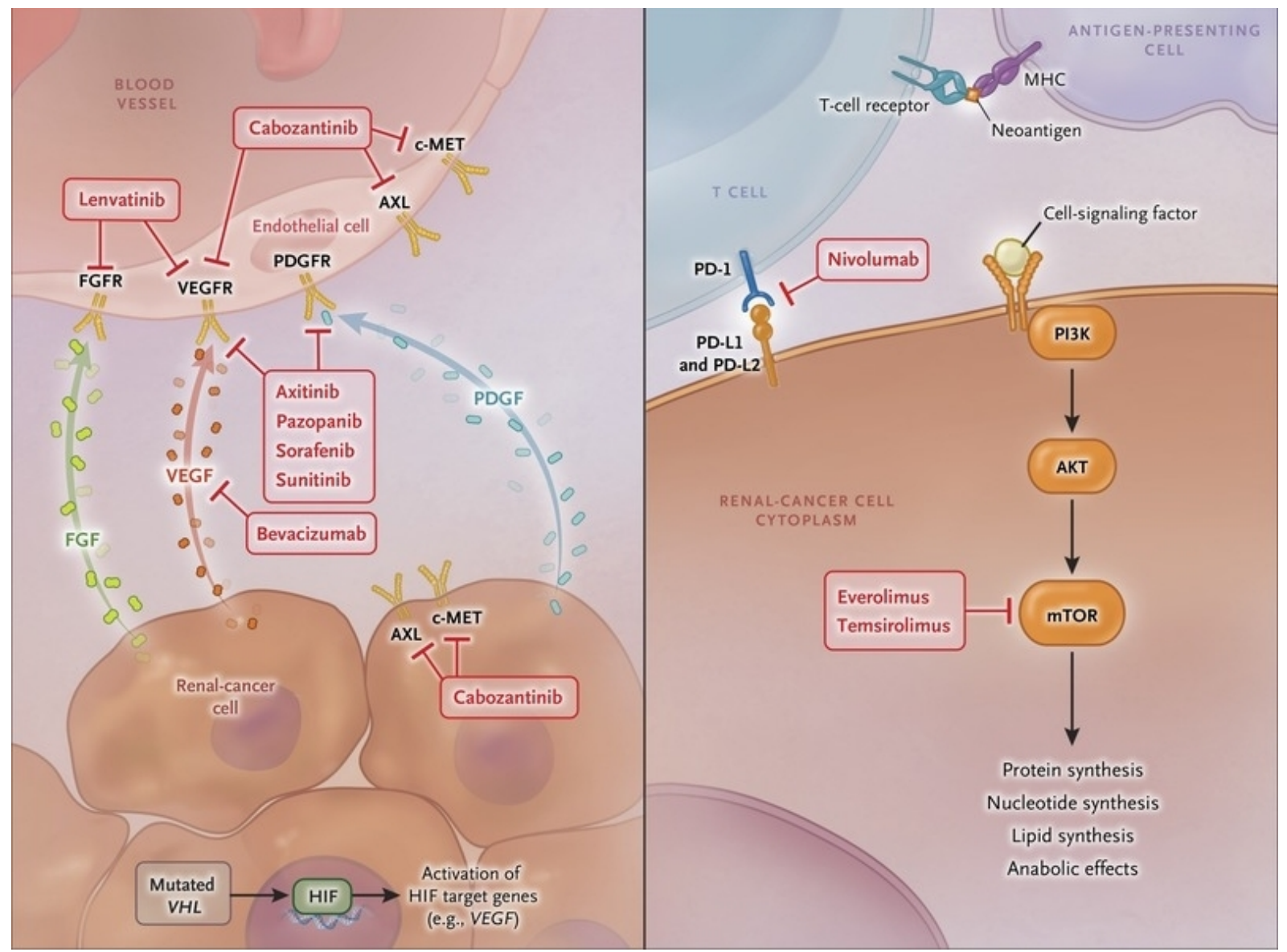

Figure 1. Metabolic pathways and the corresponding drug inhibition in mRCC $[\text { Choueiri and Motzer (2017) }]^{[46]}$.

Whole exome sequencing of metastatic ccRCC from patients treated with Nivolumab, found that PBRM1 loss was associated with a greater response to immune checkpoint therapy ${ }^{[32]}$. It has therefore been proposed that the loss of PBRM1 may alter a tumor's responsiveness to immunotherapy ${ }^{[32]}$, although this would need to be validated further before potential clinical use as a predictive biomarker.

The use of gene expression profiling has also been investigated to aid with the prognostication of RCC. Analysis implies that there are two dominant subgroups of ccRCC-type A and type $\mathrm{B}^{[33]}$. Patients with type A tumors have been found to have a significantly improved survival rate ${ }^{[33]}$. ClearCode-34 is a multigene signature model that can identify these different molecular RCC subtypes; data suggests that, when used in conjunction with the IMDC model, it can improve prognostic accuracy ${ }^{[34]}$.

\section{Non-clear cell renal cell cancer}

Approximately $25 \%$ of RCC diagnoses are of the non-clear cell subtype ${ }^{[35]}$. Non-clear cell RCC (nccRCC) consists of several different variants, each with unique histology; the most common of these is papillary RCC (pRCC) in approximately $15 \%$ of RCC diagnoses ${ }^{[35]}$. Like the clear cell, the papillary RCC is also thought to arise from the epithelium of the proximal tubule. Genomic analysis of non-clear cell tumors has identified ten notable gene mutations, including $M E T, S L C 5 A 3$ and $N F 2^{[35]}$. The Cancer Genome Atlas Network has established that there are two biologically distinct subtypes of pRCC-type 1 and type $2^{[36]}$. Type 1 tumors exhibit a greater number of MET proto-oncogene mutations and type 2 tumors are more heterogeneous in nature; although deletions of the tumor suppressor gene, CDKN2A, are associated with 
type 2 and a poorer prognosis ${ }^{[36]}$.

Investigating the efficacy of MET-directed therapies in pRCC is an area of ongoing research. A phase II trial demonstrated that the presence of a MET mutation in PRCC is highly predictive of a response to the MET inhibitor, Foretinib ${ }^{[37]}$. Furthermore, Choueiri et al. ${ }^{[38]}$ concluded an improved objective response rate (ORR) and progression-free survival (PFS) with MET-driven disease on Savolitinib, another MET inhibitor. Cabozantinib, a TKI, is already approved as a first or second-line therapy in $\mathrm{ccRCC}^{[3,40]}$; the SWOG 1500 trial is currently evaluating its specific use in pRCC due to the prevalence of MET mutations in this subtype. The results may alter the nccRCC treatment algorithm and the presence of a MET mutation could well be used as a predictive biomarker for nccRCC in the future.

Other less common nccRCC are chromophobe ( $\sim 5 \%$ of cases), oncocytoma ( $\sim 3 \%-7 \%$ cases) and collecting duct RCC $(<1 \%$ of cases), whereas sarcomatoid RCC is no longer considered as a true subtype as sarcomatoid differentiation is associated with all RCC types. Unlike ccRCC and pRCC, these rarer subtypes are thought to arise from the distal nephron, likely the epithelium of collecting tubules. Unlike in ccRCC, VHL mutation has not been found in the chromophobe subtype. However, point mutation of $p 53$ is significantly more common $(\sim 25 \%)$ as well as upregulation of KIT proto-oncogene, although gene sequencing did not show any activating point mutation ${ }^{[41-44]}$. Collecting duct RCC meanwhile shows loss of heterozygosity of chromosome 1q, 6p, 13q and $21 \mathrm{q}^{[45]}$.

\section{Circulating tumor markers}

Circulating tumor DNA (ctDNA) may accelerate biomarker discovery and provide the future solution for a more individualized approach to the management of mRCC.

Analysis has revealed extensive heterogeneity within primary RCC tumors; as such, several genetic mutations may be overlooked by obtaining only a solitary tumor biopsy. Utilizing ctDNA for testing instead provides us with a non-invasive method that could increase the likelihood of detecting genetic alterations $^{[46,47]}$.

A large study by Pal et al. ${ }^{[48]}$, which evaluated the use of ctDNA in mRCC, found that genomic alterations were identified peripherally in approximately $79 \%$ of patients; this included mutations of TP53, VHL, EGFR, NF1 and ARIDIA. They also noted ctDNA variability during different lines of therapy; there was a substantial increase in mutation frequency observed in patients on subsequent therapy, compared to firstline $\mathrm{e}^{[47]}$. This data may help broaden our understanding of therapeutic resistance.

\section{ACTIVE SURVEILLANCE IN METASTATIC RENAL CELL CARCINOMA}

The traditional approach of initiating systemic therapy immediately after diagnosing metastatic disease has been challenged in recent years. mRCC is a diverse disease with many different prognostic factors; patients with low-risk or slowly progressive malignancy may instead benefit from an initial period of active surveillance before starting systemic therapy ${ }^{[49]}$. Several trials and analyses have demonstrated that this approach may be advantageous in certain circumstances.

In 2014, Park et al. ${ }^{[49]}$ concluded that, in asymptomatic or minimally symptomatic patients, the response rates and overall survival with deferred systemic treatment were comparable to those without a surveillance period. Given the toxic and non-curative nature of therapy, this regime could positively impact a patient's quality of life. 
A similar conclusion was attained from a prospective phase II trial conducted by Rini et al. ${ }^{[50]}$. This study involved 52 asymptomatic, treatment-naïve patients who received regular radiographic follow-up throughout the surveillance period. Results suggested that deferred systemic therapy was favorable for patients with 0-1 IMDC risk factors and $<2$ organs with metastatic disease ${ }^{[50]}$.

\section{THE TREATMENT LANDSCAPE OF METASTATIC RENAL CELL CARCINOMA Cytoreductive nephrectomy}

An important element of an mRCC individualized treatment plan is the consideration that whether a cytoreductive nephrectomy $(\mathrm{CN})$ would be of benefit to the patient.

Retrospective analysis of 1658 patients, who all received targeted therapy, demonstrated that, in general, survival outcomes are substantially improved with $\mathrm{CN} v s$. without $\mathrm{CN}^{[51]}$. However, patients with a poor prognosis ( $\geq 4$ IMDC factors) may not benefit from $\mathrm{CN}^{[46]}$, and offering the procedure to this group could therefore be detrimental. Other factors to consider would be the extent of tumor burden and whether surgery is feasible.

The SURTIME randomized clinical trial investigated whether patient outcomes differed with immediate CN vs. deferred CN, after an initial period of Sunitinib before surgery ${ }^{[52]}$. Bex et al. ${ }^{[52]}$ concluded that there was no significant difference between the 28 -week progression-free rate; however, there was an improvement in overall survival with the deferred approach.

The CARMENA phase 3 trial comparing CN followed by Sunitinib, with Sunitinib alone in the setting of untreated MSKCC intermediate or poor-risk metastatic ccRCC showed that the median OS was 18.9 months with Sunitinib alone vs. 13.9 months with CN followed by Sunitinib (stratified HR for death, $0.89)^{[3,54]}$. There were no significant differences in response rate or PFS between both groups. A subsequent analysis, reclassifying patients into IMDC risk groups, demonstrated that in the intermediate-risk group in which $48.1 \%$ of patients had only one risk factor, the median OS was 30.5 months $v s .25 .2$ months in those treated with CN followed by Sunitinib vs. Sunitinib alone, respectively. For the remaining $51.9 \%$ of patients with two risk factors, the median OS was 16.6 months vs. 31.2 months with CN followed by Sunitinib vs. Sunitinib alone. Thus, the study suggested that those with either 0 or 1 IMDC risk factor should be considered for nephrectomy ${ }^{[53,54]}$.

\section{First-line systemic treatment}

In recent years, targeted therapies have supplanted cytokines in the management of mRCC, owing to a better understanding of the biological factors driving cancer growth. A new standard of care was forged in 2007 when the VEGFR-TKI, Sunitinib, outperformed Interferon-alpha in ORR (47\% vs. 12\%) and median PFS (11 months vs. 5 months) ${ }^{[55]}$. Comparison of median disease-specific survival for newly diagnosed mRCC between 1992-2004 (pre-targeted therapy era) and 2005-2009 (targeted therapy era) showed an improvement from 13 months to 16 months ${ }^{[56]}$. To date, nine targeted drugs have been approved for treating mRCC: Sunitinib, Sorafenib, Pazopanib, Cabozantinib, Tivozanib, Axitinib, Everolimus, Temsirolimus and Bevacizumab (in combination with Interferon-alpha) ${ }^{[2,57]}$.

An improved understanding of the biological immune response to cancer has led to the development of immunotherapy as a new treatment modality for mRCC. PD- 1 and CTLA- 4 are both expressed on the T cell surface, and their activation leads to a diminishing response of anti-tumor T cells. Enhanced T-cellmediated toxicity is therefore achieved by the blockade of these pathways with monoclonal antibodies against CTLA-4 (Ipilimumab) and PD-1 (Nivolumab and Pembrolizumab) or their ligand, PDL-1 
(Atezolizumab) ${ }^{[58]}$. The significant efficacy of these new immunotherapy agents in other malignancies, such as melanoma and lung cancer, ignited a burgeoning interest in using immune therapies in $\mathrm{mRCC}^{[59,60]}$. The treatment landscape for mRCC was revolutionized after the FDA approval of Nivolumab and Ipilimumab in April 2018 ${ }^{[26]}$. Since then, three additional combination therapies, Pembrolizumab plus Axitinib, Avelumab plus Axitinib, Nivolumab plus Cabozantinib, have also shown remarkable results, leading to their FDA approvals in April 2019, May 2019, and January 2021 respectively ${ }^{[61-63]}$.

\section{Favorable-risk disease}

Sunitinib has been, for many years, the preferred first-line treatment for patients identified as having favorable risk ${ }^{[64]}$. A large, randomized, phase III trial of treatment-naïve patients demonstrated that the median PFS, ORR and median OS with Sunitinib was 9.5 months, $25 \%$ and 29.3 months respectively ${ }^{[64]}$. A sub-analysis within a network meta-analysis of 15 randomized control trials showed that Sunitinib resulted in a significant PFS benefit compared with Everolimus ${ }^{[22]}$. This was likely attributable to the difference in the mechanism of action between the TKI and mTOR inhibitors. Sunitinib blocks VEGFR 1,2 and 3, as well as PDGFR $^{[17]}$. Whilst the VEGFRs may be the more pertinent targets, PDGF plays a major role in pericyte recruitment on developing tumor vessels ${ }^{[65]}$. Resistance against antiangiogenic drugs has been associated with the presence of pericyte-covered vessels ${ }^{[65]}$. As stated above, Everolimus and Temsirolimus only block the mTORC1 activation pathway, leaving mTORC2 signaling unopposed ${ }^{[23,24]}$. It has been suggested that there may be a potentially synergistic benefit from using combinations of targeted agents that inhibit separate pathways. However, a combination of Temsirolimus plus Bevacizumab, or Bevacizumab plus Interferon-alpha, only provides little survival benefit compared to Sunitinib alone, while the combination of ICI and VEGFR inhibitors as frontline therapy could provide enhanced efficacy in mRCC ${ }^{[22]}$.

In the favorable-risk group, patients treated with Ipilimumab and Nivolumab in CheckMate 214 had ORR and PFS lower than those achieved with Sunitinib $\{29 \%$ vs. 52\% and 15.3 months vs. 25.1 months [Hazard Ratio $(\mathrm{HR})=2.18 ; P<0.001]\}^{[26]}$. In ICI and VEGFR studies, a recent meta-analysis showed that Avelumab plus Axitinib is associated with a significant improvement in PFS when compared with Sunitinib ${ }^{[22,62]}$. However, additional follow-up is required in the JAVELIN Renal 101 trial to prove that this combination of results is a real OS benefit ${ }^{[62]}$. Another study, CheckMate 9ER, with Nivolumab plus Cabozantinib vs. Sunitinib, has shown benefit across all IMDC risk and PD-L1 subgroups at 18.1-month follow-up, although a longer follow-up is required to assess whether the responses are durable ${ }^{[63]}$.

The Favorable-risk disease tends to be associated with increased angiogenesis ${ }^{[6]}$. PBRM1 is the second most commonly mutated gene in ccRCC, and it plays a role in suppressing hypoxic transcriptional signatures. Its loss in the metastatic setting confers a favorable effect, potentially through the upregulation of VEGF therapy targets, such as $\mathrm{HIF}^{[67]}$.

\section{Intermediate/poor risk disease}

For patients with IMDC-identified intermediate or poor-risk disease, several different frontline regimes can be considered. The combination of ICIs with VEGFR-TKIs has emerged to be the optimal first-line therapy of choice. In this patient cohort, the CheckMate 214 trial demonstrated the superiority of Ipilimumab plus Nivolumab over Sunitinib [ORR of $42 \%$ vs. 27\% $(P<0.0001)$, PFS of 11.6 months vs. 8.4 months $(\mathrm{HR}=0.82$; 99\%CI: 0.64-1.05; $P=0.03$ ), median OS not reached vs. 26 months $]^{[26]}$. The advantage of using Avelumab plus Axitinib, over Sunitinib, in mRCC patients of all IMDC risk subgroups was demonstrated in the JAVELIN 101 trial, with an ORR of $55.2 \%$ vs. 25.7\% and a median PFS of 13.8 months vs. 8.4 months (HR = 0.69; 95\%CI: 0.56-0.84; $P=0.0001)^{[62]}$. The KEYNOTE-426 trial showed an OS benefit in favor of the Pembrolizumab plus Axitinib combination vs. Sunitinib at 18 months $(82.3 \% \text { vs. } 72.1 \%, P<0.0001)^{[6]}$. It also demonstrated an improvement in ORR and median PFS with combination therapy compared with 
Sunitinib alone $[59.3 \% \text { vs. } 35.7 \% \text { and } 15.1 \text { months vs. } 11.1 \text { months }(P<0.001) \text { respectively }]^{[61]}$. A recent Bayesian network meta-analysis suggested that the Pembrolizumab plus Axitinib combination gives optimum OS benefit for mRCC patients with intermediate and poor-risk disease ${ }^{[22]}$.

Another combination treatment study, CheckMate 9ER, with Nivolumab plus Cabozantinib vs. Sunitinib, in untreated advanced RCC, showed that at median follow-up of 18.1-month, the median PFS was 16.6 months vs. 8.3 months $(\mathrm{HR}=0.51 ; 95 \% \mathrm{CI}$ : $0.41-0.64 ; P<0.001)$ and the median $\mathrm{OS}$ was not reached $(\mathrm{HR}=$ $0.60 ; 98.89 \%$ CI: 0.40-0.89; $P=0.001)$ with benefit across all IMDC risk and PD-L1 subgroups, and ORR was $55.7 \%$ vs. $27.1 \%$ with the median duration of response of 20.2 months $v s .11 .5$ months, respectively ${ }^{[63]}$.

Recent data from the CLEAR study (study 307)/KEYNOTE-581 with a combination of Pembrolizumab plus Lenvatinib or Lenvatinib plus Everolimus $v s$. Sunitinib in the setting of advanced RCC with no prior systemic therapy showed a significantly better outcome in the combination cohorts compared to Sunitinib alone, with a median PFS of 23.9 months in the Pembrolizumab plus Lenvatinib, 15 months in the Lenvatinib plus Everolimus, and 9.2 months in Sunitinib cohort with benefit seen across all MSKCC or IMDC risk groups. Similarly, the HR for OS in Pembrolizumab plus Lenvatinib vs. Sunitinib was 0.66, with benefits seen across all MSKCC and IMDC risk groups, except for IMDC favorable risk. However, in the OS, there was no statistical difference between Lenvatinib plus Everolimus and Sunitinib, pointing to the important role of ICI in the combination strategy. The ORR was $71 \%, 54 \%$, and $36 \%$, with the complete response rate in these groups being $16 \%, 10 \%$, and $4 \%$, respectively. These results demonstrated that Pembrolizumab plus Lenvatinib is a meaningful alternative to Ipilimumab plus Nivolumab as a first-line strategy for intermediate or poor-risk patients ${ }^{[6,69]}$.

However, there is a paucity of head-to-head randomized controlled trials directly comparing the effectiveness of all available therapies. Given the variety of regimes available, it is a genuine challenge for clinicians to identify the best treatment option for each patient. Several other first-line clinical trials with combination agents (all in comparison with Sunitinib) are currently pending analyses, including Atezolizumab/Bevacizumab (IMmotion151) ${ }^{[70]}$ [Table 2].

In a patient group for which immunotherapy is contraindicated (e.g., autoimmune disease or patients on > $10 \mathrm{mg}$ baseline Prednisolone), targeted therapies can be considered as first-line. Cabozantinib is a VEGF, MET and AXL inhibitor [Figure 1], and results from the randomized phase II CABOSUN trial demonstrate improved PFS (8.2 months vs. 5.6 months) and superior ORR (46\% vs. 18\%) with Cabozantinib vs. Sunitinib respectively. In addition, patients with osseous metastatic disease receiving Cabozantinib responded better than those treated with Sunitinib ${ }^{[39]}$.

Agents inhibiting mTOR pathways, such as Temsirolimus, can be used to treat patients with the poor-risk disease. However, they are utilized less commonly than other therapies due to their limited efficacy and laborious administration schedules, which involve weekly infusions ${ }^{[66]}$. Although they can be considered for patients unable to tolerate oral drugs and those with contraindications to ICIs.

\section{Beyond first-line therapy}

A high percentage of patients with mRCC will eventually have disease progression while on first-line therapy, and switching to an alternative agent is recommended; however, the optimal therapy sequence is still an active area of research. Whilst many clinicians choose second-line VEGF-targeted therapy based on response to first-line therapy, a retrospective study of 464 patients receiving both first and second-line VEGF inhibitors demonstrated no correlation between response ${ }^{[7]]}$. 
Table 2. Selected clinical trials investigating combination therapies in the first-line setting

\begin{tabular}{|c|c|c|c|c|}
\hline Study & Treatment vs. control & $\begin{array}{l}\text { No. of } \\
\text { patients }\end{array}$ & $\begin{array}{l}\text { Comparison of median } \\
\text { PFS (months vs. } \\
\text { months) }\end{array}$ & $\begin{array}{l}\text { Comparison of OS (months } \\
\text { vs. months) }\end{array}$ \\
\hline CheckMate $214^{[26]}$ & Nivolumab/Ipilimumab vs. Sunitinib & 1096 & 11.2 vs. 8.3 & $\begin{array}{l}\text { Median OS } 48.1 \text { months vs. } \\
26.6 \text { months }(\mathrm{HR}=0.65 \\
95 \% \mathrm{Cl}: 0.54-0.78)\end{array}$ \\
\hline KEYNOTE-426 $6^{[61]}$ & Pembrolizumab/Axitinib vs. Sunitinib & 861 & 15.1 vs. 11.1 & $\begin{array}{l}\text { 12-months OS: } 89.9 \% \text { vs. } \\
78.3 \%(\mathrm{HR}=0.53 ; P<0.0001)\end{array}$ \\
\hline JAVELIN Renal-101 $^{[62]}$ & Avelumab/Axitinib vs. Sunitinib & 886 & $\begin{array}{l}13.8 \text { vs. } 7.0 \text { (in PD-L1 } \\
\text { positive population) } \\
13.3 \text { vs. } 8.0 \text { (in overall } \\
\text { population) }\end{array}$ & OS not yet reported \\
\hline IMmotion151 $^{[69]}$ & $\begin{array}{l}\text { Atezolizumab/Bevacizumab vs. } \\
\text { Sunitinib }\end{array}$ & 915 & $\begin{array}{l}11.2 \text { vs. } 7.7 \text { (in PD-L1 } \\
\text { positive population) }\end{array}$ & OS not yet reported \\
\hline CheckMate 9ER ${ }^{[63]}$ & Nivolumab/Cabozantinib vs. Sunitinib & 636 & 16.6 vs. 8.3 & OS not yet reported \\
\hline $\begin{array}{l}\text { CLEAR (Study } \\
\text { 307)/KEYNOTE-581 }{ }^{[68,69]}\end{array}$ & $\begin{array}{l}\text { Pembrolizumab/Lenvatinib vs. } \\
\text { Everolimus/Lenvatinib vs. Sunitinib }\end{array}$ & 1069 & 23.9 vs. 14.7 vs. 9.2 & OS not yet reported \\
\hline
\end{tabular}

For patients progressing on first-line ICIs, a TKI can be considered. In the phase III METEOR trial, 658 patients, previously treated with a VEGF TKI or ICI, were randomly assigned to receive Cabozantinib or Everolimus. PFS improvement was seen in the Cabozantinib group (7.4 months vs. 3.8 months) with a HR of 0.58 (95\%CI: $0.45-0.75 ; P<0.001)^{[40]}$. Another phase III study, investigating the use of Tivozanib (a novel EGFR-TKI) vs. Sorafenib in the third or fourth-line setting, showed a PFS benefit with Tivozanib across all groups, including approximately $25 \%$ of patients previously treated with VEGF-TKI/ICI combination ${ }^{[72]}$.

If a single-agent TKI is used as frontline therapy in mRCC, Nivolumab has recently been approved as a second-line option. The CheckMate 025 trial compared Nivolumab with Everolimus in 821 patients after previous antiangiogenic therapies. The study established that Nivolumab was associated with a significant improvement in OS $(25.0$ months vs. 19.6 months; $\mathrm{HR}=0.73)$ and an increased ORR $(25 \% \text { vs. } 5 \%)^{[27]}$.

The combination of Guadacitabine, a DNA hypomethylating agent, and Durvalumab, a PD-L1 inhibitor, in the setting of advanced ccRCC, has recently been investigated in a single-arm phase Ib/II trial. The first cohort consisted of patients unexposed to ICI and 0 or 1 previous treatments. Recently published data from this cohort of 42 patients, including 36 from phase II with metastatic disease, showed at a median follow-up of 20.1 months, best RECIST 1.1 response was partial response (PR) in 9 patients (22\%), stable disease (SD) in 25 patients $(61 \%)$ and progressive disease in 7 patients (17\%), with 1 non-evaluable patient. Sixty-six percent of patients derived clinical benefit, which was defined as PR or SD $\geq 6$ months with median OS not reached and median PFS being 17 months ${ }^{[73]}$.

Hypoxia-inducible factor (HIF-2 $\alpha$ ) is a transcription factor that is a key oncogenic driver in RCC. MK-6482 or Belzutifan, a first-in-class small molecule HIF-2 $\alpha$ inhibitor, has been shown to induce tumor regression in mouse xenograft RCC models. The NCT02974738 phase I/II trial investigating Belzutifan in the setting of advanced clear-cell RCC with $\geq 1$ prior therapy has shown promising results. In 55 treated patients, with a median number of prior therapies of 3, the ORR was $25 \%$, and the median PFS was 14.5 months. The disease control rates for IMDC favorable risk $(n=13)$ and intermediate or poor risk $(n=42)$ were $92 \%$ and $76 \%$, respectively ${ }^{[74]}$. 


\section{On-treatment predictive markers}

The paucity of predictive biomarkers in mRCC renders it difficult for a clinician to foresee treatment response. In recent years, it has become apparent that on-treatment predictive markers are important in determining response to therapy.

VEGF-targeted therapies e.g., Sunitinib, have common adverse effects which have been identified as potential biomarkers of clinical efficacy. These include hypertension, neutropenia and hypothyroidism ${ }^{[75]}$.

Treatment-related hypertension occurs in approximately one third of patients on Sunitinib ${ }^{[76]}$. In a large, retrospective study by Rini et al. ${ }^{[76]}$, patients with a systolic blood pressure of $\geq 140 \mathrm{mmHg}$ had a significantly improved ORR to Sunitinib, when compared with patients without systolic hypertension. PFS and OS were also longer. Further research suggests that the higher the blood pressure, the greater the systemic VEGF receptor blockade, which in turn, increases the treatment efficacy ${ }^{[77]}$. However, there is insufficient outcome data to justify changes in ongoing treatment based on these clinical biomarkers only.

\section{Oligometastatic renal cell carcinoma}

The most common sites of metastatic disease in RCC are the lung (45\%), bone (30\%), lymph nodes (22\%) and liver $(20 \%)^{[78]}$.

Sites of metastatic disease can be targeted using local therapies; this can include surgical resection (metastasectomy) and radiotherapy. These local therapies form an important part of an individualized management plan for patients with mRCC. Evidence suggests that a metastatectomy with curative intent is associated with a good long-term survival rate, particularly in younger patients with a long disease-free interval and a solitary site of metastasis ${ }^{[7]}$.

Stereotactic radiation therapy and cryotherapy are two non-invasive local therapies that are increasingly being used for oligometastatic RCC. They are also beneficial when utilized after systemic therapy to suppress residual masses ${ }^{[80]}$. Aoun et al. ${ }^{[81]}$ concluded that cryoablation is a safe and effective procedure in the management of RCC. Additionally, a study by Wang et al ${ }^{[80]}$ reported that 1-year local control rates of extracranial metastatic sites were $>90 \%$ post stereotactic radiation therapy.

Emerging data from clinical trials suggests that there may be a direct and abscopal response observed when a combination of immunotherapy and ablative therapy is used to treat $\mathrm{mRCC}^{[82]}$. However, systemic therapy is yet to be proven beneficial following complete resection of metastatic disease; the ongoing ECOG 2810 research study has, thus far, found that treatment with Pazopanib for one year after surgery does not improve chances of survival ${ }^{[83]}$.

\section{CONCLUSION}

The development of several novel therapies over recent years has revolutionized the treatment of metastatic RCC. The use of ICIs and TKIs, and their combinations, across multiple lines of therapy, have significantly improved the overall survival of patients. However, it is now becoming increasingly important for us to establish a more individualized treatment plan for mRCC, using both clinical and biological prognostic factors to guide us.

The IMDC, a clinical prognostic tool, is routinely used in practice worldwide; the model stratifies risk into three groups: favorable, intermediate and poor. Nevertheless, there is a distinct paucity of research that demonstrates any reliable predictors to therapy response. Advances in tumor genomic profiling have 
revealed potential predictive biomarkers, but further data is required before these can be used to aid clinical decision-making.

Ongoing research trials will likely continue to discover new and improved combination therapies which will further expand our capabilities of treating mRCC successfully. If we can predict who will respond to these therapies, by integrating valid biomarker data into existing prognostic tools, the development of more robust individualized RCC treatment plans will become a real possibility in the future.

\section{DECLARATIONS}

\section{Authors' contributions}

Made substantial contributions to literature review required for the manuscript as well as writing and editing it: Atmaja B, Wood I

Contributed to writing and editing the manuscript: Suyanto S, Sawhney P, Michael A, Pandha HS

Authors contributed equally.

\section{Availability of data and materials}

Not applicable.

\section{Financial support and sponsorship}

None.

\section{Conflicts of interest}

Suyanto $\mathrm{S}$ is employed by Eisai Ltd., but this review paper does not represent the view of Eisai Ltd. Suyanto $S$ is affiliated with Royal Surrey County Hospital. The remaining authors declared that there are no conflicts of interest

\section{Ethical approval and consent to participate}

Not applicable.

\section{Consent for publication}

Not applicable.

\section{Copyright}

(c) The Author(s) 2021.

\section{REFERENCES}

1. Global Cancer Observatory: Cancer Today. Lyon, France: international agency for research on cancer. Available from: https://gco.iarc.fr/today [Last accessed on 3 Jun 2021].

2. Bray F, Ferlay J, Soerjomataram I, Siegel RL, Torre LA, Jemal A. Global cancer statistics 2018: GLOBOCAN estimates of incidence and mortality worldwide for 36 cancers in 185 countries. CA Cancer J Clin 2018;68:394-424. DOI PubMed

3. Cancer research UK (2020) kidney cancer statistics. Available from: https:/www.cancerresearchuk.org/health-professional/cancerstatistics/statistics-by-cancer-type/kidney-cancer [Last accessed on 3 Jun 2021].

4. Hsieh JJ, Purdue MP, Signoretti S, et al. Renal cell carcinoma. Nat Rev Dis Primers 2017;3:17009. DOI PubMed PMC

5. Haddad AQ, Marhulis V. Tumour and patient factors in renal cell carcinoma towards personalised therapy. Nat Rev Urol 2015; 12:25362. DOI PubMed

6. Heng DYC, Xie W, Regan MM, et al. Prognostic factors for overall survival in patients with metastatic renal cell carcinoma treated with vascular endothelial growth factor-targeted agents: results from a large, multicentre study. J Clin Oncol 2009;27:5794-9. DOI PubMed

7. Motzer RJ, Bacik J, Murphy BA, et al. Interferon-alpha as a comparative treatment for clinical trials of new therapies against advanced renal cell carcinoma. J Clin Oncol 2002;20:289-96. DOI PubMed

8. Heng DYC, Xie W, Regan MM, et al. External validation and comparison with other models of the International Metastatic Renal-Cell Carcinoma Database Consortium prognostic model :a population-based study. Lancet Oncol 2013;14:141-8. DOI PubMed PMC 
9. Ko JJ, Xie W, Kroeger N, et al. The International Metastatic Renal Cell Carcinoma Database Consortium model as a prognostic tool in patients with metastatic renal cell carcinoma previously treated with first-line targeted therapy: a population-based study. Lancet Oncol 2015;16:293-300. DOI PubMed

10. Wells JC, Stukalin I, Norton C, et al. Third-line targeted therapy in metastatic renal cell carcinoma: results from the International Metastatic Renal Cell Carcinoma Database Consortium. Eur Urol 2017;71:204-9. DOI PubMed

11. Kroeger N, Xie W, Lee JL, et al. Metastatic non-clear cell renal cell carcinoma treated with targeted therapy agents: characterization of survival outcome and application of the International mRCC Database Consortium criteria. Cancer 2013;119:2999-3006. DOI PubMed PMC

12. Yip S, Wells C, Moreira RB, Wong A, Heng DYC. Real world experience of immuno-oncology agents in metastatic renal cell carcinoma: results from the IMDC. J Clin Oncol 2017;35:492.

13. Cella D, Grunwald V, Escudier B, et al. Patient-reported outcomes of patients with advanced renal cell carcinoma treated with nivolumab plus ipilimumab versus sunitinib (CheckMate 214): a randomised, phase 3 trial. Lancet Oncol 2019;20:297-310. DOI PubMed PMC

14. Srigley JR, Delahunt B, Eble JN, et al. ISUP renal tumor panel. The International Society of Urological Pathology (ISUP) vancouver classification of renal neoplasia. Am J Surg Pathol 2013;37:1469-89. DOI PubMed

15. Genome Atlas Research Network. Comprehensive molecular characterization of clear cell renal cell carcinoma. Nature 2013;499:43-9. DOI PubMed PMC

16. Patard J-J, Rioux-Leclercq N, Fergelot P. Understanding the importance of smart drugs in renal cell carcinoma. Eur Urol 2006;49:63343. DOI PubMed

17. Sternberg CN, Calabro F, Bracarda S, et al. Safety and efficacy of sunitinib in patients from Italy with metastatic renal cell carcinoma: final results form an expanded-access trial. Oncology 2015;8:273-80. DOI PubMed

18. Chowdhury B, Porter EG, Stewart JC, et al. PBRM1 regulates the expression of genes involved in metabolism and cell adhesion in renal clear cell carcinoma. PLoS One 2016;11:e0153718. DOI PubMed PMC

19. Hsieh JJ, Chen D, Wang PI, et al. Genomic biomarkers of a randomized trial comparing first-line everolimus and sunitinib in patients with metastatic renal cell carcinoma. Eur Urol 2017;71:405-14. DOI PubMed PMC

20. Carlo M, Manley B, Patil S, et al. Genomic alterations and outcomes with VEGF-targeted therapy in patients with clear cell renal cell carcinoma. Kidney Cancer 2017;1:49-56. DOI PubMed PMC

21. Creighton CJ. A gene transcription signature of the Akt/mTOR pathway in clinical breast tumors. Oncogene 2007;26:4648-55. DOI PubMed

22. Cao G, Wu X, Wang Z, et al. What is the optimum systemic treatment for advanced/metastatic renal cell carcinoma of favourable, intermediate and poor risk, respectively? BMJ Open 2020;10:eo34626. DOI PubMed PMC

23. Loewth R, Jacinto E, Wullschleger S, et al. Two TOR complexes, only one of which is rapamycin sensitive, have distinct roles in cell growth control. Mol Cell 2002;10:457-68. DOI PubMed

24. Inoki K, Guan KL. Complexity of the TOR signalling network. Trends Cell Biol 2006;16:206-12. DOI

25. Kwiatkowski DJ, Choueiri TK, Fay AP, et al. Mutations in TSC1, TSC2, and MTOR are associated with response to rapalogs in patients with metastatic renal cell carcinoma. Clin Cancer Res 2016;22:2445-52. DOI PubMed PMC

26. Motzer RJ, Tannir NM, McDermott DF, et al. Nivolumab plus Ipilimumab versus Sunitinib in advanced renal-cell carcinoma. $N$ Engl $J$ Med 2018;378:1277-90. DOI PubMed PMC

27. Motzer RJ, Escudier B, McDermott DF, et al. CheckMate 025 Investigators. Nivolumab versus everolimus in advanced renal-cell carcinoma. N Engl J Med 2015;373:1803-13. DOI PubMed PMC

28. Escudier B, Tannir NM, McDermott DF, et al. CheckMate 214: efficacy and safety of nivolumab + ipilimumab (N+I) v sunitinib (S) for treatment-naïve advanced or metastatic renal cell carcinoma (mRCC), including IMDC risk and PD-L1 expression subgroups. Ann Oncol 2017;28:V621-2. DOI

29. Mazza C, Escudier B, Albiges L. Nivolumab in renal cell carcinoma: latest evidence and clinical potential. Ther Adv Med Oncol 2017;9:171-81. DOI PubMed PMC

30. Iacovelli, R, Nole F, Verri E, et al. Prognostic role of PD-L1 expression in renal cell carcinoma. A systematic review and metaanalysis. Target Oncol 2016;11:143-48. DOI PubMed

31. RH, Dong H, Kwon ED. Implications of B7-H1 expression in clear cell carcinoma of the kidney for prognostication and therapy. Clin Cancer Res 2007;13:709s-15s.

32. Miao D, Margolis CA, Gao W, et al. Genomic correlates of response to immune checkpoint therapies in clear cell renal cell carcinoma. Science 2018;59:801-6. DOI PubMed PMC

33. Brannon AR, Reddy A, Seiler M, et al. Molecular stratification of clear cell renal cell carcinoma by consensus clustering reveals distinct subtypes and survival patterns. Genes Cancer 2010;1:152-63. DOI PubMed PMC

34. de Velasco G, Culhane AC, Fay AP, et al. Molecular subtypes improve prognostic value of International Metastatic Renal Cell Carcinoma Database Consortium prognostic model. Oncol 2017;22:286-92. DOI PubMed PMC

35. Durinck S, Stawiski EW, Pavia-Jimenez A, et al. Spectrum of diverse genomic alterations define non-clear cell renal carcinoma subtypes. Nat Genet 2015;47:13-21. DOI PubMed PMC

36. Linehan WM, Spellman PT, Ricketts CJ, et al. Cancer genome atlas research network. Comprehensive molecular characterization of papillary renal-cell carcinoma. N Engl J Med 2016;374:135-45. DOI PubMed PMC

37. Choueiri TK, Vaishampayan U, Rosenberg JE, et al. Phase II and biomarker study of the dual MET/VEGFR2 inhibitor foretinib in patients with papillary renal cell carcinoma. J Clin Oncol 2013;31:181-6. DOI PubMed PMC 
38. Choueiri TK, Plimack E, Arkenau HT, et al. Biomarker-based phase II trial of savolitinib in patients with advanced papillary renal cell cancer. J Clin Oncol 2017;35:2993-3001. DOI PubMed

39. Choueiri TK, Halabi S, Sanford BL, et al. Cabozantinib versus sunitinib as initial targeted therapy for patients with metastatic renal cell carcinoma of poor or intermediate risk: the Alliance A031203 CABOSUN trial. J Clin Oncol 2017;35:591-7. DOI PubMed PMC

40. Choueiri TK, Escudier B, Powles T, et al. METEOR Investigators. Cabozantinib versus everolimus in advanced renal-cell carcinoma. N Engl J Med 2015;373:1814-23. DOI PubMed PMC

41. Gad S, Lefevre SH, Khoo SK, et al. Mutations in BHD and TP53 genes, but not in HNF1beta gene, in a large series of sporadic chromophobe renal cell carcinoma. Br J Cancer 2007;96:336-40. DOI PubMed PMC

42. Higgins JP, Shinghal R, Gill H, et al. Gene expression patterns in renal cell carcinoma assessed by complementary DNA microarray. Am J Pathol 2003;162:925-32. DOI PubMed PMC

43. Yamazaki K, Sakamoto M, Ohta T, et al. Overexpression of KIT in chromophobe renal cell carcinoma. Oncogene 2003;22:847-52. DOI PubMed

44. Haitel A, Susani M, Wick N, et al. c-kit overexpression in chromophobe renal cell carcinoma is not associated with c-kit mutation of exons 9 and 11. Am J Surg Pathol 2005;29:842. DOI PubMed

45. Polascik TJ, Cairns P, Epstein JI, et al. Distal nephron renal tumors: microsatellite allelotype. Cancer Res 1996;56:1892-15. PubMed

46. Choueiri TK, Motzer RJ. Systemic therapy for metastatic renal-cell carcinoma. N Engl J Med 2017;376:354-66. DOI PubMed

47. Gerlinger M, Rowan AJ, Horswell S, et al. Intratumor heterogeneity and branched evolution revealed by multiregion sequencing. $N$ Engl J Med 2012;366:883-92. DOI PubMed PMC

48. Pal SK, Sonpavde G, Agarwal N, et al. Evolution of circulating tumor DNA profile from first-line to subsequent therapy in metastatic renal cell carcinoma. Eur Urol 2017;72:557-64. DOI PubMed

49. Park I, Lee JL, Ahn JH, et al. Active surveillance for metastatic or recurrent renal cell carcinoma. J Cancer Res Clin Oncol 2014;140:1421-8. DOI PubMed

50. Rini BI, Dorff TB, Elson P, et al. Active surveillance in metastatic renal-cell carcinoma: a prospective, phase 2 trial. Lancet Oncol 2016;17:1317-24. DOI PubMed

51. Heng DYC, Wells JC, Rini BI, et al. Cytoreductive nephrectomy in patients with synchronous metastases from renal cell carcinoma: results from the International Metastatic Renal Cell Carcinoma Database Consortium. Eur Urol 2014;66:704-10. DOI PubMed

52. Bex A, Mulders P, Jewett MAS, et al. Immediate versus deferred cytoreductive nephrectomy (CN) in patients with synchronous metastatic renal cell carcinoma (mRCC) receiving sunitinib (EORTC 30073 SURTIME). Ann Oncol 2017;28:V622. DOI

53. Méjean A, Ravaud A, Thezenas S, et al. Sunitinib alone or after Nephrectomy in metastatic renal-cell carcinoma. $N$ Engl J Med 2018;379:417-27. DOI PubMed

54. Méjean A, Thezenas S, Chevreau C, et al. Cytoreductive nephrectomy (CN) in metastatic renal cancer (mRCC): Update on Carmena trial with focus on intermediate IMDC-risk population. J Clin Oncol 2019;37:4508-8. DOI

55. Motzer RJ, Hutson TE, Tomczak P, et al. Overall survival and updated results for sunitinib compared with interferon alfa in patients with metastatic renal cell carcinoma. J Clin Oncol 2009;27:3584-90. DOI PubMed PMC

56. Pal SK, Nelson RA, Vogelzang N. Disease-specific survival in de novo metastatic renal cell carcinoma in the cytokine and targeted therapy era. PLoS One 2013;8:e63341. DOI PubMed PMC

57. Escudier B, Pluzanska A, Koralewski P, et al. Bevacizumanb plus interferon alfa-2a for treatment of metastatic renal cell carcinoma: a randomized, double-blind phase III trial. Lancet 2007;370(9605):2103-11. DOI PubMed

58. Ribas A, Wolchok JD. Cancer immunotherapy using checkpoint blockade. Science 2018;359:1350-5. DOI PubMed PMC

59. Hodi FS, O'Day SJ, McDermott DF, et al. Improved survival with ipilimumab in patients with metastatic melanoma. $N$ Eng $J$ Med 2010:363711-23. DOI PubMed PMC

60. Antonia SJ, López-Martin JA, Bendell J, et al. Nivolumab alone and nivolumab plus ipilimumab in recurrent small-cell lung cancer (CheckMate 032): a multicentre, open-label, phase 1/2 trial. Lancet Oncol 2016;17:883-95. DOI PubMed

61. Rini BI, Plimack ER, Stus V, et al. Pembrolizumab plus axitinib versus sunitinib for advanced renal-cell carcinoma. $N$ Engl J Med 2019;380:1103-15. DOI PubMed

62. Motzer RJ, Penkov K, Haanen J, et al. Avelumab plus axitinib versus sunitinib for advanced renal-cell carcinoma. N Engl J Med 2019;380:1103-15. DOI PubMed PMC

63. Choueiri TK, Powles T, Burotto M, et al; CheckMate 9ER Investigators. Nivolumab plus Cabozantinib versus Sunitinib for advanced renal-cell carcinoma. N Engl J Med 2021;384:829-41. DOI PubMed

64. Motzer RJ, Escudier B, Gannon A, et al. Sunitinib: ten years of successful clinical use and study in advanced renal cell carcinoma. Oncologist 2017;22:41-52. DOI PubMed PMC

65. Gee MS, Procopio WN, Makonnen S, et al. Tumor vessel development and maturation impose limits on the effectiveness of antivascular therapy. Am J Pathol 2003;162:183-93. DOI PubMed PMC

66. Kotecha RR, Motzer RJ, Voss MH. Towards individualized therapy for metastatic renal cell carcinoma. Nature 2019;16:621-33. DOI PubMed

67. Gao W, Li W, Xiao T, Liu XS, Kaelin WG Jr. Inactivation of the PBRM1 tumor suppressor gene amplifies the HIF-response in VHL/- clear cell renal carcinoma. Proc Natl Acad Sci U S A 2017;114:1027-32. DOI PubMed PMC

68. Motzer R, Alekseev B, Rha SY, et al; CLEAR Trial Investigators. Lenvatinib plus Pembrolizumab or Everolimus for advanced renal cell carcinoma. N Engl J Med 2021;384:1289-300. DOI PubMed

69. Grunwald V, Powles T, Choueiri TK, et al. Lenvatinib plus everolimus or pembrolizumab versus sunitinib in advanced renal cell carcinoma: study design and rationale. Future Oncol 2019;15:929-41. DOI PubMed 
70. Rini BI, Powles T, Atkins MB, et al. Atezolizumab plus bevacizumab versus sunitinib in patients with previously untreated metastatic renal cell carcinoma (IMmotion151): a multicentre, open-label, phase 3, randomised controlled trial. Lancet 2019;393:2404-15. DOI PubMed

71. Al-Marrawi MY, Rini BI, Harshman LC, et al. International mRCC Database Consortium. The association of clinical outcome to firstline VEGF-targeted therapy with clinical outcome to second-line VEGF-targeted therapy in metastatic renal cell carcinoma patients. Target Oncol 2013;8:203-9. DOI PubMed PMC

72. Rini BI, Pal SK, Escudier BJ, et al. Tivozanib versus sorafenib in patients with advanced renal cell carcinoma (TIVO-3): a phase 3, multicentre, randomised, controlled, open-label study. Lancet 2020;21:95-104. DOI PubMed

73. Zakharia Y, Singer EA, Ross R, Joshi M, Alva AS. Phase Ib/II study of durvalumab and guadecitabine in advanced kidney cancer Big Ten Cancer Research Consortium BTCRC GU16-043. JCO 2021;39:328. DOI

74. Choueiri TK, Bauer TM, Mcdermott DF, et al. Phase 2 study of the oral hypoxia-inducible factor $2 \alpha$ (HIF-2 $\alpha$ ) inhibitor MK-6482 in combination with cabozantinib in patients with advanced clear cell renal cell carcinoma (ccRCC). J Clin Oncol 2021;39:272. DOI

75. Kollmannsberger C. Sunitinib side effects as surrogate biomarkers of efficacy. Can Urol Assoc J 2016;10:S245-7. DOI PubMed PMC

76. Rini BI, Cohen DP, Lu DR, et al. Hypertension as a biomarker of efficacy in patients with metastatic renal cell carcinoma treated with sunitinib. J Natl Cancer Inst 2011;103:763-73. DOI PubMed PMC

77. Wasserstrum Y, Kornowski R, Raanani P, Leader A, Pasvolsky O, Iakobishvili Z. Hypertension in cancer patients treated with antiangiogenic based regimens. Cardiooncology 2015:7;1-6. DOI PubMed PMC

78. Bianchi M, Sun M, Jeldres C, et al. Distribution of metastatic sites in renal cell carcinoma: a population-based analysis. Ann Oncol 2012;23:973-80. DOI PubMed

79. Kavolius JP, Mastorakos DP, Pavlovich C, et al. Resection of metastatic renal cell carcinoma. J Clin Oncol 1998;16:2261-6. DOI PubMed

80. Wang CJ, Christie A, Lin MH, et al. Safety and efficacy of stereotactic ablative radiation therapy for renal cell carcinoma extracranial metastases. Int J Radiat Oncol Biol Phys 2017;98:91-100. DOI PubMed PMC

81. Aoun HD, Littrup PJ, Jaber M, et al. Percutaneous cryoablation of renal tumors: is it time for a new paradigm shift? J Vasc Interv Radiol 2017;28:1363-70. DOI PubMed

82. Mehta S, Illidge T, Choudhury A. Immunotherapy with radiotherapy in urological malignancies. Curr Opin Urol 2016;26:514-22. DOI PubMed

83. EurekAlert! AAAS. No benefit from Pazopanib in advanced kidney cancer after surgery to remove metastases. Available from: https://www.eurekalert.org/pub_releases/2019-06/ecrg-nbf053119.php [Last accessed on 3 Jun 2021]. 